OPEN ACCESS

Edited by:

Frederic Lamoth,

Lausanne University Hospital,

Switzerland

Reviewed by:

Oliver Kurzai

Friedrich-Schiller-University Jena,

Germany

Pierre-Yves Bochud,

Centre Hospitalier Universitaire

Vaudois, Switzerland

*Correspondence:

Cristina Cunha,

Life and Health Sciences Research Institute, School of Health Sciences,

University of Minho, Campus de Gualtar, 4710-057 Braga, Portugal cristinacunha@ecsaude.uminho.pt

Specialty section:

This article was submitted to

Fungi and Their Interactions,

a section of the journal

Frontiers in Microbiology

Received: 11 February 2015

Accepted: 20 April 2015

Published: 05 May 2015

Citation:

Oliveira-Coelho $A$,

Rodrigues F, Campos A Jr., Lacerda JF, Carvalho A and Cunha C (2015) Paving the way for predictive diagnostics and personalized treatment of invasive aspergillosis.

Front. Microbiol. 6:411.

doi: 10.3389/fmicb.2015.00411

\section{Paving the way for predictive diagnostics and personalized treatment of invasive aspergillosis}

\author{
Ana Oliveira-Coelho ${ }^{1,2}$, Fernando Rodrigues ${ }^{1,2}$, António Campos Jr. ${ }^{3}$, João F. Lacerda ${ }^{4,5}$, \\ Agostinho Carvalho ${ }^{1,2}$ and Cristina Cunha ${ }^{1,2 *}$ \\ 1 Life and Health Sciences Research Institute (ICVS), School of Health Sciences, University of Minho, Braga, \\ Portugal, ${ }^{2}$ ICVS/3B'S - PT Government Associate Laboratory, Braga/Guimarães, Portugal, ${ }^{3}$ Serviço de Transplantação de \\ Medula Óssea, Instituto Português de Oncologia do Porto, Porto, Portugal, ${ }^{4}$ Instituto de Medicina Molecular, Faculdade de \\ Medicina de Lisboa, Lisboa, Portugal, ${ }^{5}$ Serviço de Hematologia e Transplantação de Medula, Hospital de Santa Maria, \\ Lisboa, Portugal
}

Invasive aspergillosis (IA) is a life-threatening fungal disease commonly diagnosed among individuals with immunological deficits, namely hematological patients undergoing chemotherapy or allogeneic hematopoietic stem cell transplantation. Vaccines are not available, and despite the improved diagnosis and antifungal therapy, the treatment of IA is associated with a poor outcome. Importantly, the risk of infection and its clinical outcome vary significantly even among patients with similar predisposing clinical factors and microbiological exposure. Recent insights into antifungal immunity have further highlighted the complexity of host-fungus interactions and the multiple pathogen-sensing systems activated to control infection. How to decode this information into clinical practice remains however, a challenging issue in medical mycology. Here, we address recent advances in our understanding of the host-fungus interaction and discuss the application of this knowledge in potential strategies with the aim of moving toward personalized diagnostics and treatment (theranostics) in immunocompromised patients. Ultimately, the integration of individual traits into a clinically applicable process to predict the risk and progression of disease, and the efficacy of antifungal prophylaxis and therapy, holds the promise of a pioneering innovation benefiting patients at risk of IA.

Keywords: invasive aspergillosis, stem cell transplantation, antifungal immunity, single nucleotide polymorphism, personalized medicine

\section{Introduction}

Aspergillosis includes an extensive spectrum of diseases caused by fungi of the genus Aspergillus with clinical manifestations that range from colonization to allergic bronchopulmonary aspergillosis and disseminated forms of infection (Segal, 2009). The prevalence of invasive aspergillosis (IA) has steadily increased in the last decades, mostly due to the advent of solid organ and hematopoietic stem cell transplantation (HSCT), and the increased use of chemotherapy and immunosuppression (Kontoyiannis et al., 2010; Pagano et al., 2010). Although the diagnosis of IA has improved, namely because of the introduction of biomarkers such as the detection of galactomannan in the clinical practice (Morrissey et al., 2013), successful treatment is still a challenging endeavor. Indeed, established infection is difficult to eradicate, resulting in associated mortality rates ranging from 40 to $90 \%$ (Walsh et al., 2008). 
The extensive use of empiric antifungal therapy is a major concern given the potential development of resistance in the pathogen. The emergence of azole-resistant strains of Aspergillus fumigatus (Steinmann et al., 2015) underscores the need for personalized diagnostic and risk prediction approaches based on individual traits that may allow targeting antifungal therapy and improving outcomes without unnecessary empiric therapy. Several host genetic variants have been proposed as suitable markers to categorize patients with the highest risk of infection (Cunha et al., 2013). However, many challenges confront the development of these predictive tools. Among them, our insufficient understanding of the critical immune defects that predispose to the infection and the lack of validation of genetic diagnostics in well-designed clinical trials.

The purpose of this review is to reposition novel findings on the host-fungus interaction and to discuss the challenges in exploiting this knowledge to the design of patient-tailored diagnostic or therapeutic approaches to deliver to specific target populations.

\section{The Host-fungus Interaction: What's New?}

The physical barriers afforded by the respiratory mucosa confer the first line of innate resistance to fungal infection. Because of their small size, conidia may reach the lung alveoli, where they are phagocytosed and killed through the production of reactive oxygen species (ROS) by resident macrophages, whereas neutrophils are instead proficient at handling hyphae germinating from conidia that escape macrophage surveillance through an array of extracellular killing mechanisms (Ibrahim-Granet et al., 2003; Cunha et al., 2014b; Heinekamp et al., 2015). This morphotype preference may be in part due to the ability of neutrophils to sense microbe size and selectively release neutrophil extracellular traps in response to larger structures (Branzk et al., 2014). Importantly, recent findings also suggest that lung epithelial cells act as an active extension of the innate immune system, operating as a surveillance mechanism sensing fungal spores and prompting antifungal effector responses (Osherov, 2012).

Innate immune cells are equipped with pattern recognition receptors (PRRs) able to discriminate pathogen-associated molecular patterns (PAMPs; Bourgeois and Kuchler, 2012; Plato et al., 2015). Stimulation of antigen-presenting cells, including macrophages and dendritic cells (DCs), leads to the activation and recruitment of lymphocytes, and the development of adaptive antifungal immune responses. Once committed, T cells monitor the host for infection and mobilize appropriate effector functions by inducing cytokines and cytolytic molecules, which are instrumental in rallying and activating professional phagocytes to the site of microbial deposition, thus providing a prompt and effective control of infection (Romani, 2011).

The fungal cell wall is the main source of PAMPs owing to its dynamic composition and structural properties according to morphotype, growth stage and environmental conditions (Latge, 2010). Toll-like receptor (TLR)-2 (in cooperation with TLR1 and TLR6), TLR3, TLR4, and TLR9, and the C-type lectin receptors dectin-1, dendritic cell-specific intercellular adhesion molecule 3 grabbing non-integrin (DC-SIGN) and mannose receptor are the most important PRRs recognizing fungal PAMPs including mannan, $\beta$-glucan and nucleic acids (Romani, 2011).

\section{The Host Perspective}

The immune response to A. fumigatus is determined not only by the relative degree of stimulation of the individual PRRs but also by the level of receptor cooperation and cellular localization. Indeed, a sequential activation of distinct signal transduction pathways through the PRR adapters myeloid differentiation primary response $88(\mathrm{MyD} 88)$ and caspase-associated recruitment domain 9 (CARD9) in the respiratory epithelium and hematopoietic compartment in response to infection was recently reported (Jhingran et al., 2015). By means of a partial overlap, these signals ensure optimal chemokine induction, neutrophil recruitment, and fungal clearance within the respiratory tract.

Patients undergoing immunosuppressive regimens based on calcineurin inhibitors such as cyclosporine A or corticosteroids are highly susceptible to disseminated fungal infections. Previous work demonstrated that calcineurin is an important regulator of dectin-1-mediated signaling and activation of immunity to Candida albicans (Greenblatt et al., 2010), and that corticosteroid immunosuppression blocks dectin-1-mediated signaling required for maturation of A. fumigatus phagosomes (Kyrmizi et al., 2013). More recently, the calcineurin inhibitor tacrolimus was found to impair primary alveolar macrophage activation in response to $A$. fumigatus by preventing a signaling pathway involving a TLR9Bruton's tyrosine kinase-calcineurin-nuclear factor of activated T-cells axis required for proinflammatory cytokine production (Herbst et al., 2015). Taken together, these findings suggest that disseminated fungal infections seen in these patients are not just a general consequence of systemic suppression of adaptive immunity but are, rather, a result of the specific blockade of evolutionarily conserved innate pathways for fungal resistance.

Another important feature of dectin-1-mediated signaling in response to A. fumigatus is the activation of the nucleotidebinding oligomerization domain-like receptor family, pyrin domain containing 3 (NLRP3) inflammasome leading to the production of bioactive interleukin (IL)-1 $\beta$ (Said-Sadier et al., 2010). Indeed, members of the IL-1 receptor family of cytokines are critical effector molecules in antifungal immunity (Gresnigt and van de Veerdonk, 2014). By preventing activation of the NLRP3 inflammasome and reducing IL- $1 \beta$ secretion, IL-37 was recently found to act as a broad spectrum inhibitor of innate responses to fungal infection-mediated inflammation (Moretti et al., 2014). Along this line, the increased IL- $1 \beta$ release intrinsically associated to chronic granulomatous disease (CGD) was found to be reverted by the use of IL-1 receptor antagonist (IL-1Ra), leading to restrained neutrophil recruitment and $\mathrm{T}$ helper 17 responses, thereby protecting from IA (de Luca et al., 2014).

Soluble PRRs found in the fluids lining the epithelial surfaces support fungal sensing by binding to conidia and enhancing their uptake by phagocytes. Among these, the long pentraxin 3 (PTX3) was demonstrated to play a non-redundant role in antifungal host defense (Garlanda et al., 2002) by enhancing recognition and phagocytosis through mechanisms that depend on $\mathrm{Fc} \gamma$ receptor, CD11b and complement activation (Moalli et al., 2010). Engagement of myeloid differentiation protein 2 (MD-2) during uptake 
of PTX3-opsonized conidia was also revealed to activate TLR4 signaling converging on the production of type I interferons (Bozza et al., 2014). This suggests that in addition to pro-phagocytic properties, PTX3 is able to elicit antifungal effector mechanisms associated with limited immunopathology, thereby highlighting potential mechanisms of action underlying its favorable synergism with antifungal therapy against IA (Lo Giudice et al., 2012; Marra et al., 2014).

\section{The Pathogen Perspective}

Fungi have evolved their own elaborate mechanisms to escape innate immunity. By masking dectin-1 and dectin-2-dependent recognition, the hydrophobin layer of A. fumigatus conidia restrains neutrophil infiltration and cytokine production (Aimanianda et al., 2009; Carrion Sde et al., 2013). Galactosaminogalactan (GAG), a polysaccharide of the fungal cell wall produced by glucose epimerases (Lee et al., 2014), also hampers neutrophil recruitment (Fontaine et al., 2011). GAG functions as an adhesin, mediating adherence and suppressing host inflammatory responses, in part through masking cell wall $\beta$-glucan from recognition by dectin-1 (Gravelat et al., 2013). The immunosuppressive properties of GAG have also been attributed to its potent ability to induce IL-1Ra (Gresnigt et al., 2014), a finding further highlighting a possible therapeutic option targeting IL-1Ra in IA.

Genomic and transcriptomic approaches have revealed that fungal pathogenicity depends also on mechanisms regulating fungal metabolism and response to stress in adaptation to the host environment. In particular, the ability of A. fumigatus to adapt to hypoxic microenvironments has been found to involve the production of secondary metabolites that promote lung inflammation, exacerbate infection and influence subsequent host immune responses (Grahl et al., 2011). Given the need of myeloid cells to adapt to hypoxic and inflamed microenvironments that develop during infection, the hypoxia-inducible factor 1-alpha (HIF-1 $\alpha$ ) has been found to be essentially required for chemokine production and maintenance of neutrophil numbers in the lungs of infected animals (Shepardson et al., 2014).

Effector T cell responses are also targeted by fungi. For example, mucosal vaccination was found to subvert $\mathrm{T}$ cell priming by impairing chemokine signals on egress of inflammatory monocytes from the bone marrow and their recruitment to the lung (Wuthrich et al., 2012). This finding is even more significant considering the role of inflammatory monocytes in orchestrating antifungal immunity in the lung by regulating the conidiocidal activity of neutrophils and their own differentiation to DCs (Espinosa et al., 2014). Importantly, inflammatory monocytes are required for optimal IL-1 $\alpha$ expression in the lungs, which in turn regulates the early accumulation of neutrophils in the lung (Caffrey et al., 2015).

\section{Host Genetic Determinants of Risk of IA}

The inborn deficiency of the phagocyte nicotinamide adenine dinucleotide phosphate (NADPH) oxidase leading to a defective production of ROS and underlying CGD is the best known example of primary immunodeficiency associated with a distinctive predisposition to IA (Vinh, 2011). Patients with autosomal-dominant
hyper-IgE syndrome (AD-HIES) are also at risk for IA (Vinh et al., 2010); however, the susceptibility of AD-HIES patients classically results from concurrent anatomical lung defects from previous bacterial infections and defective STAT3-dependent epithelial immunity (Holland et al., 2007). For most individuals however, the genetic propensity to IA has a polygenic source that acts in combination with other remarkable predisposing variants (e.g., the profound immunosuppression typical of many clinical settings), and that may translate into further immunological dysfunction, ultimately increasing the proneness to infection.

Our increasing ability to analyze human variability at the DNA level has made possible the identification of genetic factors implicated in the development of IA in hematological patients (Cunha et al., 2013), providing important insights into the mechanisms of human diversity underlying increased susceptibility to IA. Although genetic profiling is nowadays regarded as a promising methodology to exploit in the future toward improved diagnosis and therapy of fungal diseases (Cunha and Carvalho, 2012), this field is still bedeviled by difficulties, mostly related to heterogeneity of cohorts, sample size, selection bias, and statistical flaws (van der Velden et al., 2011), which compromise the clinical applicability of this knowledge.

The identification of a donor haplotype in TLR 4 increasing risk of IA after HSCT was one of the first solid reports illustrating the remarkable influence of host genetics on the susceptibility to IA (Bochud et al., 2008). This phenotype was associated with a delayed $\mathrm{T}$ cell and natural killer $\mathrm{T}$ cell immune reconstitution after transplant (Koldehoff et al., 2013). However, despite TLR4 polymorphisms have also been linked with chronic aspergillosis in immunocompetent individuals (Carvalho et al., 2008) and fungal colonization in HSCT recipients (Carvalho et al., 2009), their prognostic significance remains unclear, since the exact mechanism(s) through which TLR4 deficiency impacts human antifungal immunity remain to be identified. Along this line, the early finding that genetic variants in TLR1 and TLR6 predisposed to IA among HSCT recipients (Kesh et al., 2005) was recently supported by evidence demonstrating the defective production of crucial antifungal cytokines and chemokines by TLR1 - and TLR6deficient mouse cells after stimulation with A. fumigatus (Rubino et al., 2012).

The discovery that sensing of fungal RNA by TLR3 was required for the activation of protective memory $\mathrm{CD} 8^{+} \mathrm{T}$ cells responses in experimental aspergillosis was complemented by the identification of a regulatory variant impairing the expression of the human receptor in human DCs and hampering the efficient priming of memory $\mathrm{CD}^{+}$responses (Carvalho et al., 2012b). These findings suggest that, by interpreting immunogenetic signatures and identifying subtle differences in immune profiles, response efficiencies to potential antifungal vaccination strategies are likely to be discriminated. In fact, the measurement of A. fumigatus-specific immune responses in hematological patients was confirmed as a promising immunodiagnostic approach (Potenza et al., 2013) amenable to combination with other diagnostic tools. Finally, a stop codon in recipient TLR5 was also disclosed as an important prognostic factor for the development of IA among HSCT recipients (Grube et al., 2013), a finding warranting further studies into the function of this receptor in antifungal immunity. 
Dectin-1 deficiency was also consistently reported to contribute to susceptibility to IA (Cunha et al., 2010; Chai et al., 2011; Sainz et al., 2012). The finding that by compromising the surface expression and dectin-1-mediated cytokine production, the presence of the Y238X polymorphism in HSCT donors and recipients displayed a cumulative effect toward risk for infection (Cunha et al., 2010) emphasizes the contribution of non-hematopoietic dectin-1 to antifungal immunity. In addition, although damage perception is fundamental for resolution of fungal infection (Cunha et al., 2012), genetic variants triggering hyperactive danger signaling, and presumably leading to uncontrolled inflammatory responses to the fungus, were also recently found to increase risk for IA (Cunha et al., 2011).

A number of positive associations between genetic variants in cytokine and chemokine genes and vulnerability to IA has also been reported (Sainz et al., 2007a,b, 2008, 2010; Mezger et al., 2008; Carvalho et al., 2010). One recent example regards the identification of polymorphisms in the genes encoding for IL-1 $\beta$ and beta-defensin 1 (DEFB1) that, by affecting production of $A$. fumigatus-induced proinflammatory cytokines by mononuclear cells, influenced susceptibility to mold infection after solid organ transplantation (Wojtowicz et al., 2014).

A number of unconventional strategies have been employed to uncover additional candidate genes for susceptibility to IA (Zaas et al., 2008; Durrant et al., 2011). For example, genetic mapping analysis of survival data of infected mice allowed the identification of plasminogen, a regulatory molecule with opsonic properties, as a fitting contestant for susceptibility (Zaas et al., 2008). Consequently, a non-synonymous polymorphism in human plasminogen was found to increase risk for IA in HSCT recipients. Genetic and functional deficiency of other molecules with opsonic activity-e.g., mannose-binding lectin (MBL; Lambourne et al., 2009) and PTX3 (Cunha et al., 2014a) - has also been disclosed as a major determinant of susceptibility to IA, pointing to a key contribution of innate humoral responses to antifungal immunity. Indeed, a donor haplotype in PTX3 associated

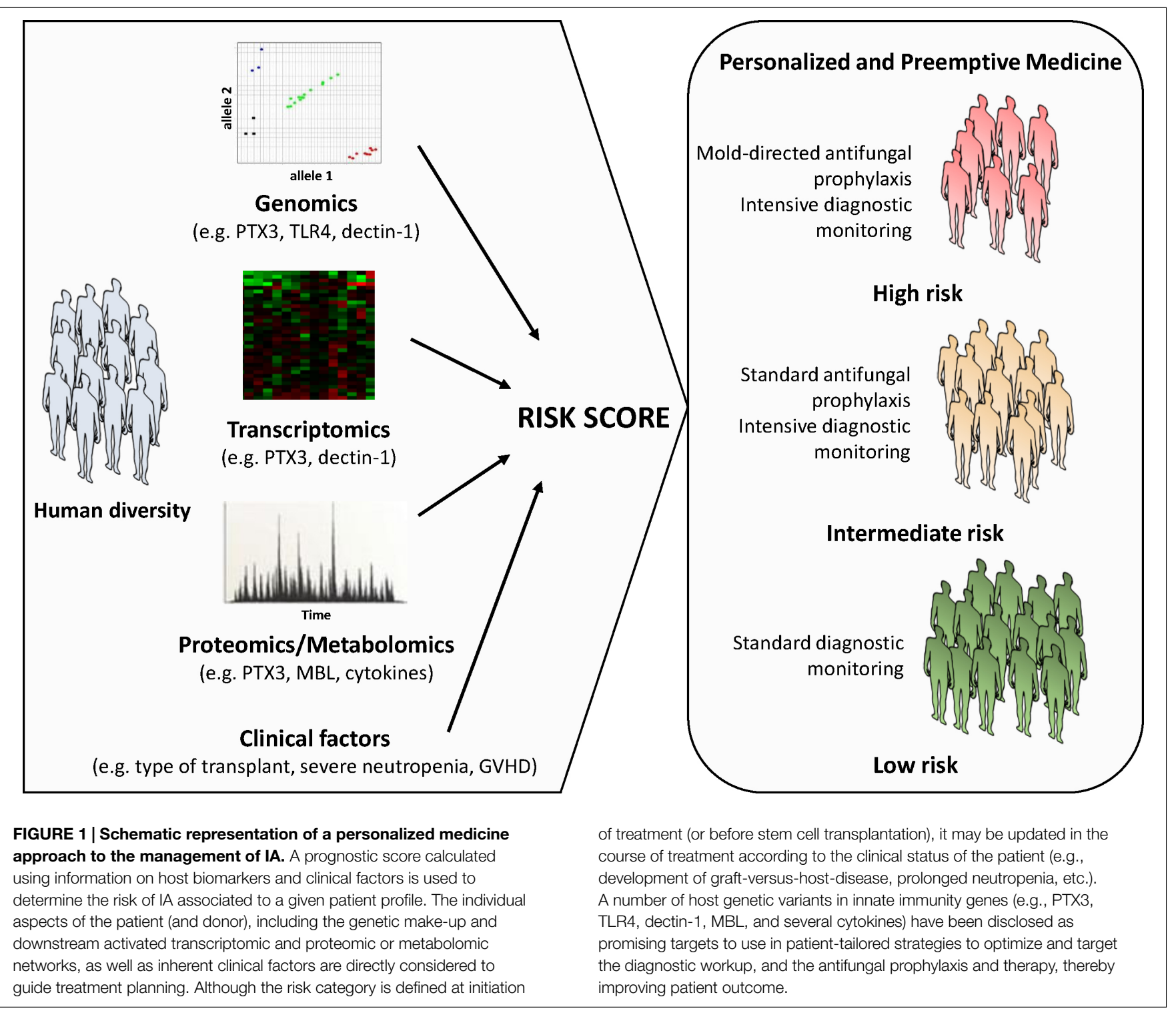


with increased risk of IA in the corresponding HSCT recipient was found to compromise PTX3 expression during the developmental programming of neutrophils in the bone marrow, leading to a defective antifungal capacity of newly reconstituted neutrophils (Cunha et al., 2014a). Importantly, this association was recently replicated in a cohort of solid organ transplant recipients (Wojtowicz and Bochud, 2015). The fact that exogenous administration of PTX3 is able to revert the genetic defect (Cunha et al., 2014a) further highlights the potential of PTX3-based immunotherapies to treat (or prevent) IA (Carvalho et al., 2012a).

\section{Decoding the Host-fungus Interaction into Clinical Strategies}

Early diagnosis is crucial to a favorable outcome of IA. However, the existing diagnostic tools are often compromised by slowness, invasiveness, lack of standardization and insufficient understating of their kinetics (Hope et al., 2005). The introduction of molecular and serological diagnostic techniques into clinical practice has undoubtedly improved our capacity to diagnose IA. Nonetheless, the broad applicability of both techniques is hampered by considerable variability in performance. Given these technical barriers, the search for tools to diagnose IA that are more efficient and reliable is an active field of research. One example was recently provided by a study demonstrating the usefulness of direct detection of exogenous fungal metabolites in the breath to the identification of the underlying microbial etiology of pneumonia (Koo et al., 2014).

Although the interaction of the fungus with the immune system is being exploited to project novel and improved fungal diagnostics, efforts have on the other hand been also devoted to the implementation of clinical models aimed at the prediction of infection in high-risk patients. In this regard, interpretation of individual genomic, transcriptomic, proteomic or metabolomic profiles associated with impaired antifungal immune responses and their integration with clinical data is regarded as a promising approach (Figure 1). Indeed, next-generation sequencing technologies now provide exciting possibilities to pin down essential steps in host-fungus interaction at a level of complexity previously unanticipated. The first genome-wide association studies

\section{References}

Aimanianda, V., Bayry, J., Bozza, S., Kniemeyer, O., Perruccio, K., Elluru, S. R., et al. (2009). Surface hydrophobin prevents immune recognition of airborne fungal spores. Nature 460, 1117-1121. doi: 10.1038/nature08264

Bochud, P. Y., Chien, J. W., Marr, K. A., Leisenring, W. M., Upton, A., Janer, M., et al. (2008). Toll-like receptor 4 polymorphisms and aspergillosis in stemcell transplantation. N. Engl. J. Med. 359, 1766-1777. doi: 10.1056/NEJMoa 0802629

Bourgeois, C., and Kuchler, K. (2012). Fungal pathogens-a sweet and sour treat for toll-like receptors. Front. Cell Infect. Microbiol. 2:142. doi: 10.3389/fcimb.2012.00142

Bozza, S., Campo, S., Arseni, B., Inforzato, A., Ragnar, L., Bottazzi, B., et al. (2014). PTX3 binds MD-2 and promotes TRIF-dependent immune protection in aspergillosis. J. Immunol. 193, 2340-2348. doi: 10.4049/jimmunol.1400814

Branzk, N., Lubojemska, A., Hardison, S. E., Wang, Q., Gutierrez, M. G., Brown, G. D., et al. (2014). Neutrophils sense microbe size and selectively release neutrophil extracellular traps in response to large pathogens. Nat. Immunol. 15, 1017-1025. doi: 10.1038/ni.2987
(GWAS) exploring host susceptibility to IA are underway and are expected to provide unbiased insights into the genetic defects contributing to development of IA, thereby laying the foundation for clinical trials aimed at the validation of medical interventions based on individual genomics. These efforts are nonetheless centered on the fairly "static" role played by the genetic variants. Physiological responses to fungal infection require the coordinated regulation of gene expression, which may vary markedly between individuals and influence phenotypes such as protein levels, the cell morphology and function, and ultimately the immunity to infection. Thus, genetic analysis of molecular traits such as the gene expression represents a powerful approach enabling insights into the human genomic landscape by generating expression maps useful for the functional interpretation of non-coding variants likely to arise from ongoing genome-wide initiatives (Fairfax and Knight, 2014).

\section{Conclusions and Perspectives}

The discovery of accurate and reliable genetic markers of susceptibility may be a turning point toward innovative stratification strategies based on genetic screening or immune profiling to predict risk and severity of disease, efficacy of antifungal prophylaxis and therapy, and eventually contribute to the successful design of antifungal vaccines. As shown for PTX3 deficiency (Cunha et al., 2014a), targeting cell function (e.g., exogenous administration of lacking or deficient factors) may prove an interesting approach to be validated in the future. Indeed, engineering of T cell function to target carbohydrates was demonstrated as a potentially exploitable strategy for the treatment of IA (Kumaresan et al., 2014). Ultimately, approaches based on individual genomics (and with influence on multiple functional transcriptomic, proteomic and metabolomic networks) may warrant important clinical tools allowing discrimination of patients that require enhanced surveillance for fungal disease or alternative antifungal therapies.

\section{Acknowledgement}

CC is supported by the Fundação para a Ciência e Tecnologia, Portugal (SFRH/BPD/96176/2013).

Caffrey, A. K., Lehmann, M. M., Zickovich, J. M., Espinosa, V., Shepardson, K M., Watschke, C. P., et al. (2015). IL-1alpha signaling is critical for leukocyte recruitment after pulmonary Aspergillus fumigatus challenge. PLoS Pathog. 11:e1004625. doi: 10.1371/journal.ppat.1004625

Carrion Sde, J., Leal, S. M. Jr., Ghannoum, M. A., Aimanianda, V., Latge, J. P., and Pearlman, E. (2013). The RodA hydrophobin on Aspergillus fumigatus spores masks dectin-1- and dectin-2-dependent responses and enhances fungal survival in vivo. J. Immunol. 191, 2581-2588. doi: 10.4049/jimmunol.1300748

Carvalho, A., Cunha, C., Bistoni, F., and Romani, L. (2012a). Immunotherapy of aspergillosis. Clin. Microbiol. Infect. 18, 120-125. doi: 10.1111/j.14690691.2011.03681.x

Carvalho, A., De Luca, A., Bozza, S., Cunha, C., D’angelo, C., Moretti, S., et al. (2012b). TLR3 essentially promotes protective class I-restricted memory CD8 ${ }^{+}$ T-cell responses to Aspergillus fumigatus in hematopoietic transplanted patients. Blood 119, 967-977. doi: 10.1182/blood-2011-06-362582

Carvalho, A., Cunha, C., Carotti, A., Aloisi, T., Guarrera, O., Di Ianni, M., et al. (2009). Polymorphisms in Toll-like receptor genes and susceptibility to infections in allogeneic stem cell transplantation. Exp. Hematol. 37, 1022-1029. doi: 10.1016/j.exphem.2009.06.004 
Carvalho, A., Cunha, C., Di Ianni, M., Pitzurra, L., Aloisi, T., Falzetti, F., et al. (2010). Prognostic significance of genetic variants in the IL-23/Th17 pathway for the outcome of T cell-depleted allogeneic stem cell transplantation. Bone Marrow Transplant. 45, 1645-1652. doi: 10.1038/bmt.2010.28

Carvalho, A., Pasqualotto, A. C., Pitzurra, L., Romani, L., Denning, D. W., and Rodrigues, F. (2008). Polymorphisms in toll-like receptor genes and susceptibility to pulmonary aspergillosis. J. Infect. Dis. 197, 618-621. doi: 10.1086/526500

Chai, L. Y., De Boer, M. G., Van Der Velden, W. J., Plantinga, T. S., Van Spriel, A. B., Jacobs, C., et al. (2011). The Y238X stop codon polymorphism in the human beta-glucan receptor dectin-1 and susceptibility to invasive aspergillosis. J. Infect. Dis. 203, 736-743. doi: 10.1093/infdis/jiq102

Cunha, C., Aversa, F., Lacerda, J. F., Busca, A., Kurzai, O., Grube, M., et al. (2014a). Genetic PTX3 deficiency and aspergillosis in stem-cell transplantation. N. Engl. J. Med. 370, 421-432. doi: 10.1056/NEJMoa1211161

Cunha, C., Kurzai, O., Loffler, J., Aversa, F., Romani, L., and Carvalho, A. (2014b). Neutrophil responses to aspergillosis: new roles for old players. Mycopathologia 178, 387-393. doi: 10.1007/s11046-014-9796-7

Cunha, C., Aversa, F., Romani, L., and Carvalho, A. (2013). Human genetic susceptibility to invasive aspergillosis. PLoS Pathog. 9:e1003434. doi: 10.1371/journal.ppat.1003434

Cunha, C., and Carvalho, A. (2012). Host genetics and invasive fungal diseases: towards improved diagnosis and therapy? Expert Rev. Anti Infect Ther. 10, 257-259. doi: 10.1586/eri.12.3

Cunha, C., Carvalho, A., Esposito, A., Bistoni, F., and Romani, L. (2012). DAMP signaling in fungal infections and diseases. Front. Immunol. 3:286. doi: 10.3389/fimmu.2012.00286

Cunha, C., Di Ianni, M., Bozza, S., Giovannini, G., Zagarella, S., Zelante, T., et al. (2010). Dectin-1 Y238X polymorphism associates with susceptibility to invasive aspergillosis in hematopoietic transplantation through impairment of both recipient- and donor-dependent mechanisms of antifungal immunity. Blood 116, 5394-5402. doi: 10.1182/blood-2010-04-279307

Cunha, C., Giovannini, G., Pierini, A., Bell, A. S., Sorci, G., Riuzzi, F., et al. (2011). Genetically-determined hyperfunction of the S100B/RAGE axis is a risk factor for aspergillosis in stem cell transplant recipients. PLOS ONE 6:e27962. doi: 10.1371/journal.pone.0027962

de Luca, A., Smeekens, S. P., Casagrande, A., Iannitti, R., Conway, K. L., Gresnigt, M. S., et al. (2014). IL-1 receptor blockade restores autophagy and reduces inflammation in chronic granulomatous disease in mice and in humans. Proc. Natl. Acad. Sci. U.S.A. 111, 3526-3531. doi: 10.1073/pnas.1322831111

Durrant, C., Tayem, H., Yalcin, B., Cleak, J., Goodstadt, L., De Villena, F. P., et al. (2011). Collaborative Cross mice and their power to map host susceptibility to Aspergillus fumigatus infection. Genome Res. 21, 1239-1248. doi: 10.1101/gr.118786.110

Espinosa, V., Jhingran, A., Dutta, O., Kasahara, S., Donnelly, R., Du, P., et al. (2014). Inflammatory monocytes orchestrate innate antifungal immunity in the lung. PLoS Pathog. 10:e1003940. doi: 10.1371/journal.ppat.1003940

Fairfax, B. P., and Knight, J. C. (2014). Genetics of gene expression in immunity to infection. Curr. Opin. Immunol. 30C, 63-71. doi: 10.1016/j.coi.2014.07.001

Fontaine, T., Delangle, A., Simenel, C., Coddeville, B., Van Vliet, S. J., Van Kooyk, Y., et al. (2011). Galactosaminogalactan, a new immunosuppressive polysaccharide of Aspergillus fumigatus. PLoS Pathog. 7:e1002372. doi: 10.1371/journal.ppat.1002372

Garlanda, C., Hirsch, E., Bozza, S., Salustri, A., De Acetis, M., Nota, R., et al. (2002). Non-redundant role of the long pentraxin PTX3 in anti-fungal innate immune response. Nature 420, 182-186. doi: 10.1038/nature01195

Grahl, N., Puttikamonkul, S., Macdonald, J. M., Gamcsik, M. P., Ngo, L. Y., Hohl, T. M., et al. (2011). In vivo hypoxia and a fungal alcohol dehydrogenase influence the pathogenesis of invasive pulmonary aspergillosis. PLoS Pathog. 7:e1002145. doi: 10.1371/journal.ppat.1002145

Gravelat, F. N., Beauvais, A., Liu, H., Lee, M. J., Snarr, B. D., Chen, D., et al. (2013). Aspergillus galactosaminogalactan mediates adherence to host constituents and conceals hyphal beta-glucan from the immune system. PLoS Pathog. 9:e1003575. doi: 10.1371/journal.ppat.1003575

Greenblatt, M. B., Aliprantis, A., Hu, B., and Glimcher, L. H. (2010). Calcineurin regulates innate antifungal immunity in neutrophils. J. Exp. Med. 207, 923-931. doi: 10.1084/jem.20092531

Gresnigt, M. S., Bozza, S., Becker, K. L., Joosten, L. A., Abdollahi-Roodsaz, S., Van Der Berg, W. B., et al. (2014). A polysaccharide virulence factor from Aspergillus fumigatus elicits anti-inflammatory effects through induction of
Interleukin-1 receptor antagonist. PLoS Pathog. 10:e1003936. doi: 10.1371/journal.ppat.1003936

Gresnigt, M. S., and van de Veerdonk, F. L. (2014). The role of interleukin-1 family members in the host defence against Aspergillus fumigatus. Mycopathologia 178, 395-401. doi: 10.1007/s11046-014-9776-y

Grube, M., Loeffler, J., Mezger, M., Kruger, B., Echtenacher, B., Hoffmann, P., et al. (2013). TLR5 stop codon polymorphism is associated with invasive aspergillosis after allogeneic stem cell transplantation. Med. Mycol. 51, 818-825. doi: 10.3109/13693786.2013.809630

Heinekamp, T., Schmidt, H., Lapp, K., Pahtz, V., Shopova, I., Koster-Eiserfunke, N., et al. (2015). Interference of Aspergillus fumigatus with the immune response. Semin. Immunopathol. 37, 141-152. doi: 10.1007/s00281-014-0465-1

Herbst, S., Shah, A., Mazon Moya, M., Marzola, V., Jensen, B., Reed, A., et al. (2015). Phagocytosis-dependent activation of a TLR9-BTK-calcineurin-NFAT pathway co-ordinates innate immunity to Aspergillus fumigatus. EMBO Mol. Med. 7, 240-258. doi: 10.15252/emmm.201404556

Holland, S. M., Deleo, F. R., Elloumi, H. Z., Hsu, A. P., Uzel, G., Brodsky, N., et al. (2007). STAT3 mutations in the hyper-IgE syndrome. N. Engl. J. Med. 357, 1608-1619. doi: 10.1056/NEJMoa073687

Hope, W. W., Walsh, T. J., and Denning, D. W. (2005). Laboratory diagnosis of invasive aspergillosis. Lancet Infect. Dis. 5, 609-622. doi: 10.1016/S14733099(05)70238-3

Ibrahim-Granet, O., Philippe, B., Boleti, H., Boisvieux-Ulrich, E., Grenet, D., Stern, M., et al. (2003). Phagocytosis and intracellular fate of Aspergillus fumigatus conidia in alveolar macrophages. Infect. Immun. 71, 891-903. doi: 10.1128/IAI.71.2.891-903.2003

Jhingran, A., Kasahara, S., Shepardson, K. M., Junecko, B. A., Heung, L. J., Kumasaka, D. K., et al. (2015). Compartment-specific and sequential role of MyD88 and CARD9 in chemokine induction and innate defense during respiratory fungal infection. PLoS Pathog. 11:e1004589. doi: 10.1371/journal.ppat.1004589

Kesh, S., Mensah, N. Y., Peterlongo, P., Jaffe, D., Hsu, K., VAN DEN Brink, M., et al. (2005). TLR1 and TLR6 polymorphisms are associated with susceptibility to invasive aspergillosis after allogeneic stem cell transplantation. Ann. N. Y. Acad. Sci. 1062, 95-103. doi: 10.1196/annals.1358.012

Koldehoff, M., Beelen, D. W., and Elmaagacli, A. H. (2013). Increased susceptibility for aspergillosis and post-transplant immune deficiency in patients with gene variants of TLR4 after stem cell transplantation. Transpl. Infect. Dis. 15, 533-539. doi: $10.1111 /$ tid. 12115

Kontoyiannis, D. P., Marr, K. A., Park, B. J., Alexander, B. D., Anaissie, E. J., Walsh, T. J., et al. (2010). Prospective surveillance for invasive fungal infections in hematopoietic stem cell transplant recipients, 2001-2006: overview of the Transplant-Associated Infection Surveillance Network (TRANSNET) Database. Clin. Infect. Dis. 50, 1091-1100. doi: 10.1086/651263

Koo, S., Thomas, H. R., Daniels, S. D., Lynch, R. C., Fortier, S. M., Shea, M. M., et al. (2014). A breath fungal secondary metabolite signature to diagnose invasive aspergillosis. Clin. Infect. Dis. 59, 1733-1740. doi: 10.1093/cid/ciu725

Kumaresan, P. R., Manuri, P. R., Albert, N. D., Maiti, S., Singh, H., Mi, T., et al. (2014). Bioengineering $\mathrm{T}$ cells to target carbohydrate to treat opportunistic fungal infection. Proc. Natl. Acad. Sci. U.S.A. 111, 10660-10665. doi: 10.1073/pnas.1312789111

Kyrmizi, I., Gresnigt, M. S., Akoumianaki, T., Samonis, G., Sidiropoulos, P., Boumpas, D., et al. (2013). Corticosteroids block autophagy protein recruitment in Aspergillus fumigatus phagosomes via targeting dectin-1/Syk kinase signaling. J. Immunol. 191, 1287-1299. doi: 10.4049/jimmunol.1300132

Lambourne, J., Agranoff, D., Herbrecht, R., Troke, P. F., Buchbinder, A., Willis, F., et al. (2009). Association of mannose-binding lectin deficiency with acute invasive aspergillosis in immunocompromised patients. Clin. Infect. Dis. 49, 1486-1491. doi: $10.1086 / 644619$

Latge, J. P. (2010). Tasting the fungal cell wall. Cell. Microbiol. 12, 863-872. doi: 10.1111/j.1462-5822.2010.01474.x

Lee, M. J., Gravelat, F. N., Cerone, R. P., Baptista, S. D., Campoli, P. V., Choe, S. I., et al. (2014). Overlapping and distinct roles of Aspergillus fumigatus UDP-glucose 4-epimerases in galactose metabolism and the synthesis of galactose-containing cell wall polysaccharides. J. Biol. Chem. 289, 1243-1256. doi: $10.1074 /$ jbc.M113.522516

Lo Giudice, P., Campo, S., De Santis, R., and Salvatori, G. (2012). Effect of PTX3 and voriconazole combination in a rat model of invasive pulmonary aspergillosis. Antimicrob. Agents Chemother. 56, 6400-6402. doi: 10.1128/AAC.01000-12 
Marra, E., Sousa, V. L., Gaziano, R., Pacello, M. L., Arseni, B., Aurisicchio, L., et al. (2014). Efficacy of PTX3 and posaconazole combination in a rat model of invasive pulmonary aspergillosis. Antimicrob. Agents Chemother. 58, 6284-6286. doi: 10.1128/AAC.03038-14

Mezger, M., Steffens, M., Beyer, M., Manger, C., Eberle, J., Toliat, M. R., et al. (2008). Polymorphisms in the chemokine ( $\mathrm{C}-\mathrm{X}-\mathrm{C}$ motif) ligand 10 are associated with invasive aspergillosis after allogeneic stem-cell transplantation and influence CXCL10 expression in monocyte-derived dendritic cells. Blood 111, 534-536. doi: 10.1182/blood-2007-05-090928

Moalli, F., Doni, A., Deban, L., Zelante, T., Zagarella, S., Bottazzi, B., et al. (2010). Role of complement and Fc $\gamma$ receptors in the protective activity of the long pentraxin PTX3 against Aspergillus fumigatus. Blood 116, 5170-5180. doi: 10.1182/blood-2009-12-258376

Moretti, S., Bozza, S., Oikonomou, V., Renga, G., Casagrande, A., Iannitti, R. G., et al. (2014). IL-37 inhibits inflammasome activation and disease severity in murine aspergillosis. PLoS Pathog. 10:e1004462. doi: 10.1371/journal.ppat.1004462

Morrissey, C. O., Chen, S. C., Sorrell, T. C., Milliken, S., Bardy, P. G., Bradstock, K. F., et al. (2013). Galactomannan and PCR versus culture and histology for directing use of antifungal treatment for invasive aspergillosis in high-risk haematology patients: a randomised controlled trial. Lancet Infect. Dis. 13, 519-528. doi: 10.1016/S1473-3099(13)70076-8

Osherov, N. (2012). Interaction of the pathogenic mold Aspergillus fumigatus with lung epithelial cells. Front. Microbiol. 3:346. doi: 10.3389/fmicb.2012.00346

Pagano, L., Caira, M., Candoni, A., Offidani, M., Martino, B., Specchia, G., et al. (2010). Invasive aspergillosis in patients with acute myeloid leukemia: a SEIFEM-2008 registry study. Haematologica 95, 644-650. doi: 10.3324/haematol.2009.012054

Plato, A., Hardison, S. E., and Brown, G. D. (2015). Pattern recognition receptors in antifungal immunity. Semin Immunopathol. 37, 97-106. doi: 10.1007/s00281014-0462-4

Potenza, L., Vallerini, D., Barozzi, P., Riva, G., Forghieri, F., Beauvais, A., et al. (2013). Characterization of specific immune responses to different Aspergillus antigens during the course of invasive Aspergillosis in hematologic patients. PLoS ONE 8:e74326. doi: 10.1371/journal.pone.0074326

Romani, L. (2011). Immunity to fungal infections. Nat. Rev. Immunol. 11, 275-288. doi: $10.1038 /$ nri2939

Rubino, I., Coste, A., Le Roy, D., Roger, T., Jaton, K., Boeckh, M., et al. (2012). Species-specific recognition of Aspergillus fumigatus by Toll-like receptor 1 and Toll-like receptor 6. J. Infect. Dis. 205, 944-954. doi: 10.1093/infdis/ jir882

Said-Sadier, N., Padilla, E., Langsley, G., and Ojcius, D. M. (2010). Aspergillus fumigatus stimulates the NLRP3 inflammasome through a pathway requiring ROS production and the Syk tyrosine kinase. PLOS ONE 5:e10008. doi: 10.1371/journal.pone.0010008

Sainz, J., Hassan, L., Perez, E., Romero, A., Moratalla, A., Lopez-Fernandez, E., et al. (2007a). Interleukin-10 promoter polymorphism as risk factor to develop invasive pulmonary aspergillosis. Immunol. Lett. 109, 76-82. doi: 10.1016/j.imlet.2007.01.005

Sainz, J., Perez, E., Hassan, L., Moratalla, A., Romero, A., Collado, M. D., et al. (2007b). Variable number of tandem repeats of TNF receptor type 2 promoter as genetic biomarker of susceptibility to develop invasive pulmonary aspergillosis. Hum. Immunol. 68, 41-50. doi: 10.1016/j.humimm.2006. 10.011

Sainz, J., Lupianez, C. B., Segura-Catena, J., Vazquez, L., Rios, R., Oyonarte, S., et al. (2012). Dectin-1 and DC-SIGN polymorphisms associated with invasive pulmonary Aspergillosis infection. PLOS ONE 7:e32273. doi: 10.1371/journal.pone. 0032273
Sainz, J., Perez, E., Gomez-Lopera, S., and Jurado, M. (2008). IL1 gene cluster polymorphisms and its haplotypes may predict the risk to develop invasive pulmonary aspergillosis and modulate C-reactive protein level. J. Clin. Immunol. 28, 473-485. doi: 10.1007/s10875-008-9197-0

Sainz, J., Salas-Alvarado, I., Lopez-Fernandez, E., Olmedo, C., Comino, A., Garcia, F., et al. (2010). TNFR1 mRNA expression level and TNFR1 gene polymorphisms are predictive markers for susceptibility to develop invasive pulmonary aspergillosis. Int. J. Immunopathol. Pharmacol. 23, 423-436.

Segal, B. H. (2009). Aspergillosis. N. Engl. J. Med. 360, 1870-1884. doi: 10.1056/NEJMra0808853

Shepardson, K. M., Jhingran, A., Caffrey, A., Obar, J. J., Suratt, B. T., Berwin, B. L., et al. (2014). Myeloid derived hypoxia inducible factor 1-alpha is required for protection against pulmonary Aspergillus fumigatus infection. PLoS Pathog. 10:e1004378. doi: 10.1371/journal.ppat.1004378

Steinmann, J., Hamprecht, A., Vehreschild, M. J., Cornely, O. A., Buchheidt, D., Spiess, B., et al. (2015). Emergence of azole-resistant invasive aspergillosis in HSCT recipients in Germany. J. Antimicrob. Chemother. 70, 1522-1526. doi: $10.1093 / \mathrm{jac} / \mathrm{dku} 566$

van der Velden, W. J., Blijlevens, N. M., and Donnelly, J. P. (2011). Genetic variants and the risk for invasive mould disease in immunocompromised hematology patients. Curr. Opin. Infect. Dis. 24, 554-563. doi: 10.1097/QCO. 0b013e32834ab1f4

Vinh, D. C. (2011). Insights into human antifungal immunity from primary immunodeficiencies. Lancet Infect. Dis. 11, 780-792. doi: 10.1016/S1473-3099(11) 70217-1

Vinh, D. C., Sugui, J. A., Hsu, A. P., Freeman, A. F., and Holland, S. M. (2010). Invasive fungal disease in autosomal-dominant hyper-IgE syndrome. J. Allergy Clin. Immunol. 125, 1389-1390. doi: 10.1016/j.jaci.2010.01.047

Walsh, T. J., Anaissie, E. J., Denning, D. W., Herbrecht, R., Kontoyiannis, D. P., Marr, K. A., et al. (2008). Treatment of aspergillosis: clinical practice guidelines of the Infectious Diseases Society of America. Clin. Infect. Dis. 46, 327-360. doi: $10.1086 / 525258$

Wojtowicz, A., and Bochud, P. Y. (2015). Host genetics of invasive Aspergillus and Candida infections. Semin Immunopathol. 37, 173-86. doi: 10.1007/s00281-0140468-y

Wojtowicz, A., Gresnigt, M. S., Lecompte, T., Bibert, S., Manuel, O., Joosten, L. A., et al. (2014). IL1B and DEFB1 polymorphisms increase susceptibility to invasive mold infection after solid-organ transplantation. J. Infect. Dis. doi: 10.1093/infdis/jiu636 [Epub ahead of print].

Wuthrich, M., Ersland, K., Sullivan, T., Galles, K., and Klein, B. S. (2012) Fungi subvert vaccine $\mathrm{T}$ cell priming at the respiratory mucosa by preventing chemokine-induced influx of inflammatory monocytes. Immunity 36, 680-692. doi: 10.1016/j.immuni.2012.02.015

Zaas, A. K., Liao, G., Chien, J. W., Weinberg, C., Shore, D., Giles, S. S., et al. (2008). Plasminogen alleles influence susceptibility to invasive aspergillosis. PLoS Genet. 4:e1000101. doi: 10.1371/journal.pgen.1000101

Conflict of Interest Statement: The authors declare that the research was conducted in the absence of any commercial or financial relationships that could be construed as a potential conflict of interest.

Copyright (C) 2015 Oliveira-Coelho, Rodrigues, Campos Jr., Lacerda, Carvalho and Cunha. This is an open-access article distributed under the terms of the Creative Commons Attribution License (CC BY). The use, distribution or reproduction in other forums is permitted, provided the original author(s) or licensor are credited and that the original publication in this journal is cited, in accordance with accepted academic practice. No use, distribution or reproduction is permitted which does not comply with these terms. 